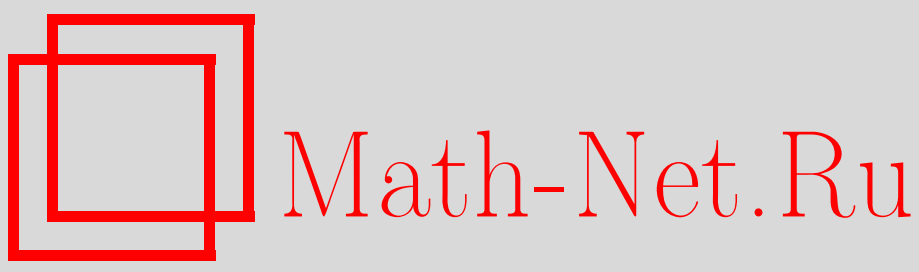

Вик. С. Куликов, В. М. Харламов, О вещественных структурах на жестких поверхностях, Изв. РАН. Сер. матем., 2002, том 66, выпуск 1, 133-152

DOI: https://doi.org/10.4213/im374

Использование Общероссийского математического портала Math-Net.Ru подразумевает, что вы прочитали и согласны с пользовательским соглашением http://www. mathnet.ru/rus/agreement

Параметры загрузки:

IP : 3.85 .7 .115

26 апреля 2023 г., $12: 44: 47$ 
УДК $512.7+515.1$

Вик. С. Куликов, В.М. Харламов

\title{
О вещественных структурах на жестких поверхностях
}

\begin{abstract}
Построены примеры жестких поверхностей (т.е. поверхностей, деформационные классы которых состоят из одной поверхности), ведущих себя по-разному по отношению к вещественным структурам: в одном из примеров поверхность не имеет вещественной структуры, а в другом - имеет единственную вещественную структуру, которая не является максимальной относительно неравенства Смита-Тома. Таким образом, эти примеры дают отрицательные решения следующих проблем: существование вещественных поверхностей в каждом деформационном классе комплексных поверхностей и существование максимальной вещественной поверхности в каждом деформационном классе, содержащем вещественную поверхность. Кроме того, доказано, что среди поверхностей основного типа с $p_{g}=q=0$ и $K^{2}=9$ нет вещественных.

Построенные поверхности дают новые контрпримеры к "Dif=Def"-проблеме.

Библиография: 14 наименований.
\end{abstract}

\section{Введение}

Типичная постановка задач вещественной алгебраической геометрии заключается в следуюшем: фиксируется деформационный класс комплексных многообразий и в этом классе изучаются те многобразия, которые могут быть снабжены вешественной структурой, и затем исследуются их топологические, а также другие, инвариантные относительно вещественных деформаций, свойства. Особенно интересны те многообразия, которые являются максимальными относительно неравенства Смита-Тома, так как они обладают замечательными топологическими свойствами (см., например, обзор [3]); для поверхностей это неравенство приведено в $\S 5$. Таким образом, возникают два естественных вопроса: в каждом ли деформационном классе комплексных компактных многообразий содержится вешественное многообразие?; и в каждом ли комплексном деформационном классе, содержащем вещественные многообразия, найдется максимальное вещественное многообразие? Насколько известно, в размерности $\geqslant 2$ оба эти вопроса до сих пор оставались открытыми. Покажем, что ответы на эти вопросы отрищательны.

В наших примерах многообразия - это жесткие поверхности, где жесткость означает, что пространство модулей комплексных структур на подлежащем гладком многообразии является 0-мерным. Более того, в наших примерах поверхности являются сильно жесткими, т.е. факторпространство пространства модулей этих поверхностей по действию канонического комплексного сопряжения (меняющего

Работа была выполнена во время пребывания первого автора в Страсбургском университете и частично поддержана грантами INTAS-97-2072, NWO-RFBR-047-008-005 и РФФИ, № 99-01-01133.

(C) Вик.С. Куликов, В.М. ХАРлАмов, 2002 
комплексную структуру поверхности на комплексно-сопряженную и, тем самым, голоморфные функции - на антиголоморфные; ориентация подлежашего гладкого четырехмерного многообразия при этом не меняется) является одной точкой. Стоит отметить, что в первом из наших примеров пространство модулей состоит из двух сопряженных точек, а во втором примере оно сводится к одной вешественной точке (см. замечания в $\S 4$ ). Кроме того, две сопряженные поверхности из первого примера дают еще один контрпример к "Dif=Def"-проблеме (первые контрпримеры к которой были построены Манетти [9]). Во всех наших примерах поверхности - это поверхности основного типа с $c_{1}^{2}=3 c_{2}$ (так называемые поверхности Мияоки-Яо; утверждения о том, что они являются сильно жесткими и, более того, что эти поверхности определяются своим гомотопическим типом однозначно с точностью до голомофных и антиголоморфных диффеоморфизмов, общеизвестны; см., например, [1]). Следуя работе [5], мы строим такие жесткие поверхности, как (конечные абелевы) накрытия Галуа (раздутой) проективной плоскости, разветвленные вдоль некоторых конфигураций прямых. В $\S 1,2$ приведена исчерпьвающая конструкция таких поверхностей в виде факторпространств накрытия Ферма. В $\S 3$ изучается группа автоморфизмов и антиавтоморфизмов построенных поверхностей. В $\S 4$ приведены три основных примера. В $\S 5$ мы рассматриваем фальшивые проективные плоскости (т.е. поверхности основного типа с $c_{2}=3$ и $c_{1}^{2}=9$ ). Доказано, что они не имеют антиголоморфных диффеоморфизмов и, в частности, не могут быть снабжены вешественной структурой. Этот параграф̆ содержит также несколько замечаний, относящихся к смежным темам.

Авторы благодарны И. Мияоке, привлекшего наш интерес к вещественной геометрии жестких поверхностей, и Т. Дельзану за полезные предложения во время подготовки данной статьи к публикации. Второй автор благодарен также $\Phi$. Катанезе за интересное обсуждение деформационных проблем вещественной алгебраической геометрии.

\section{§ 1. Накрытия Галуа плоскости, разветвленные над конфигурацией прямых}

По определению накрытие Галуа гладкого алгебраического многообразия $Y$ это конечный морфизм $f: X \rightarrow Y$ нормального алгебраического многообразия $X$ на $Y$ такой, что вложение полей рациональных функций $\mathbb{C}(Y) \subset \mathbb{C}(X)$, индуцированное морфизмом $f$, является расширением Галуа. Как хорошо известно, конечный морфизм $f: X \rightarrow Y$ является накрытием Галуа с группой Галуа $G$ тогда и только тогда, когда $G$ совпадает с группой накрьвающих преобразований, действующих транзитивно на каждом слое морфизма $f$. Кроме того, конечное разветвленное накрытие является накрытием Галуа тогда и только тогда, когда неразветвленная часть этого накрытия (т.е. ограничение морфизма на дополнения к ветвлениям внизу и наверху) является накрытием Галуа. Более того, разветвленное накрытие Галуа определяется с точностью до изоморфизма своей неразветвленной частью и морфизм накрытий из неразветвленной части одного разветвленного накрытия в неразветвленную часть другого может быть продолжен до морфизма разветвленных накрытий, если задано продолжение морфизма накрываемых многообразий в точки ветвления накрытия. Напомним также, что неразветвленное накрытие является накрытием Галуа с группой Галуа $G$ тогда и только тогда, когда 
это топологическое накрытие ассощиировано с эпиморфизмом из фундаментальной группы накрываемого многообразия в $G$ и, в частности, накрытия Галуа с абелевой группой Галуа $G$ взаимно однозначно соответствуют эпиморфизмам из первой группы гомологий с целыми коэффициентами накрываемого многообразия в $G$. Все перечисленные свойства накрытий Галуа являются хорошо известными, и наиболее нетривиальная часть из них может быть получена, например, из теоремы Грауэрта-Ремерта [4] (детальное описание основных свойств разветвленных накрытий можно найти, например, в [12]).

В дальнейшем мы будем иметь дело только с накрытиями комплексной проективной плоскости $\mathbb{P}^{2}$, разветвленными вдоль некоторой конфигурации прямых $L=L_{1} \cup \cdots \cup L_{n}$. Подобно общим абелевым накрытиям Галуа, абелево накрытие Галуа $g: Y \rightarrow \mathbb{P}^{2}$ с абелевой группой Галуа $G$, разветвленное вдоль $L$, однозначно определяется некоторым эпиморфизмом $\varphi: H_{1}\left(\mathbb{P}^{2} \backslash L, \mathbb{Z}\right) \rightarrow G$ и сушествует для любого такого эпиморфизма. Так как $H_{1}\left(\mathbb{P}^{2} \backslash L, \mathbb{Z}\right) \simeq \mathbb{Z}^{n-1}$, то существует, в частности, накрытие $g_{u(m)}: Y_{u(m)} \rightarrow \mathbb{P}^{2}$, соответствуюшее естественному эпиморфизму

$$
\varphi: H_{1}\left(\mathbb{P}^{2} \backslash L, \mathbb{Z}\right) \rightarrow H_{1}\left(\mathbb{P}^{2} \backslash L, \mathbb{Z} / m \mathbb{Z}\right)=H_{1}\left(\mathbb{P}^{2} \backslash L, \mathbb{Z}\right) \otimes(\mathbb{Z} / m \mathbb{Z}) .
$$

Назовем его накрытием Ферма. Следующее утверждение является непосредственным следствием обших результатов о разветвленных накрытиях, упомянутых в начале этого параграфа.

ПРЕДЛОЖЕНИЕ 1.1. Если $g: Y \rightarrow \mathbb{P}^{2}$ является накрытием Галуа с әруппой Галуа $G \simeq(\mathbb{Z} / m \mathbb{Z})^{k}$, разветвленным вдоль $L$, то $k \leqslant n-1$ и для любого эпиморфизма $H_{1}\left(\mathbb{P}^{2} \backslash L\right) \rightarrow G$ существует однозначно определенное накрытие Галуа $f: Y_{u(m)} \rightarrow Y$, индуцированное этим эпиморфизмом и такое, что $g_{u(m)}=g \circ f$.

Ниже будем иметь дело только с накрытиями Галуа с группой Галуа $G \simeq$ $(\mathbb{Z} / m \mathbb{Z})^{k}$ и будем строить их так, как это описано в приведенном выше предложении.

Простые петли $\lambda_{i}, 1 \leqslant i \leqslant n$, вокруг прямых $L_{i}$ порождают $H_{1}\left(\mathbb{P}^{2} \backslash L, \mathbb{Z}\right) \simeq$ $\mathbb{Z}^{n-1}$. Эти петли удовлетворяют соотношению

$$
\lambda_{1}+\cdots+\lambda_{n}=0
$$

и без ограничения обшности мы можем предполагать, что накрытие $е$ ерма $g_{u(m)}$ : $Y_{u(m)} \rightarrow \mathbb{P}^{2}$ определяется эпиморфизмом $\varphi: H_{1}\left(\mathbb{P}^{2} \backslash L, \mathbb{Z}\right) \rightarrow(\mathbb{Z} / m \mathbb{Z})^{n-1}$, отображающим $\lambda_{n}$ в $(m-1, \ldots, m-1)$ и $\lambda_{i}, 1 \leqslant i \leqslant n-1$, в $(0, \ldots, 0,1,0, \ldots, 0)$, где 1 стоит на $i$-ом месте. Выберем дополнительную прямую $L_{\infty} \subset \mathbb{P}^{2}$, находящуюся в обшем положении по отношению к конфигурации прямых $L$, и введем аффинные координаты $\left(x_{1}, x_{2}\right)$ в $\mathbb{C}^{2}=\mathbb{P}^{2} \backslash L_{\infty}$. Пусть $l_{i}\left(x_{1}, x_{2}\right)=0$ - линейное уравнение прямой $L_{i} \cap \mathbb{C}^{2}$. Положим $z_{i}=\left(l_{i} l_{n}^{m-1}\right)^{1 / m}, 1 \leqslant i \leqslant n-1$. Тогда поле рациональных функций $K_{u(m)}=\mathbb{C}\left(Y_{u(m)}\right)=\mathbb{C}\left(x_{1}, x_{2}, z_{1}, \ldots, z_{n-1}\right)$ на $Y_{u(m)}$ является абелевым расширением поля рациональных фунций $k=\mathbb{C}\left(x_{1}, x_{2}\right)$ на $\mathbb{P}^{2}$ степени $m^{n-1}$ с группой Галуа

$$
G=\left\{\gamma=\left(\gamma_{1}, \ldots, \gamma_{n}\right) \in(\mathbb{Z} / m \mathbb{Z})^{n} \mid \sum_{i=1}^{n} \gamma_{i} \equiv 0 \quad(\bmod m)\right\} \simeq(\mathbb{Z} / m \mathbb{Z})^{n-1} .
$$


(Другими словами, прообраз $\mathbb{P}^{2} \backslash L_{\infty}$ в $Y_{u(m)}$ изоморфен нормализации аффинного подмногообразия в $\mathbb{C}^{n+1}$, заданного в координатах $x_{1}, x_{2}, z_{1}, \ldots, z_{n-1}$ уравнениями $z_{1}^{m}=l_{1} l_{n}^{m-1}, \ldots, z_{n-1}^{m}=l_{n-1} l_{n}^{m-1}$.)

Положим

$$
z^{a}=\prod_{i=1}^{n-1} z_{i}^{\alpha_{i}}
$$

для мультииндекса $a=\left(\alpha_{1}, \ldots, \alpha_{n-1}\right), 0 \leqslant \alpha_{i} \leqslant m-1$. Действие элемента $\gamma=\left(\gamma_{1}, \ldots, \gamma_{n}\right) \in G$ на $K_{u(m)}$ задается формулой

$$
\gamma\left(z^{a}\right)=\mu^{(\gamma, a)} z^{a}
$$

где

$$
(\gamma, a)=\sum_{j=1}^{n-1} \gamma_{j} \alpha_{j}
$$

и $\mu=e^{2 \pi i / m}-$ корень $m$-ой степени из единицы. Таким образом,

$$
K_{u(m)}=\bigoplus_{0 \leqslant \alpha_{i} \leqslant m-1} \mathbb{C}\left(x_{1}, x_{2}\right) z^{a}
$$

является разложением векторного пространства $K_{u(m)}$ над $\mathbb{C}\left(x_{1}, x_{2}\right)$ в конечную прямую сумму степени 1 представлений группы $G$.

Пусть $\varphi: H_{1}\left(\mathbb{P}^{2} \backslash L, \mathbb{Z}\right) \rightarrow(\mathbb{Z} / m \mathbb{Z})^{k}$ - эпиморфизм, заданный на образуюших формулами $\varphi\left(\lambda_{i}\right)=\left(\alpha_{i, 1}, \ldots, \alpha_{i, k}\right)$, где $\alpha_{1, j}+\cdots+\alpha_{n, j} \equiv 0 \bmod m$ для каждого $j=1, \ldots, k$, и пусть $g: Y \rightarrow \mathbb{P}^{2}$ - соответствуюшее накрытие Галуа. Тогда согласно предложению 1.1 существует единственное накрытие Галуа $f: Y_{u(m)} \rightarrow Y$. Оно определяет вложение $f^{*}: \mathbb{C}(Y) \rightarrow K_{u(m)}$ поля рациональных функций $\mathbb{C}(Y)$ на $Y$ в поле рациональных функций $K_{u(m)}=\mathbb{C}\left(Y_{u(m)}\right)$. Ясно, что $\mathbb{C}(Y)$ совпадает с подполем $K_{\varphi}=\mathbb{C}\left(x_{1}, x_{2}, w_{1}, \ldots, w_{k}\right)$ поля $K_{u(m)}$, где $w_{j}=z_{1}^{\alpha_{1, j}} \ldots z_{n-1}^{\alpha_{n-1, j}}$, и

$$
\operatorname{Gal}\left(K_{u(m)} / K_{\varphi}\right)=\left\{\left(\gamma_{1}, \ldots, \gamma_{n}\right) \in G \mid \sum_{i=1}^{n-1} \alpha_{i, j} \gamma_{i} \equiv 0 \bmod m, 1 \leqslant j \leqslant k\right\}
$$

По построению $Y$ является нормальной поверхностью с изолированными особыми точками. Особые точки поверхности $Y$ могут возникнуть только над $r$-кратными точками конфигурации $L$ с $r \geqslant 2$, т.е. над точками, лежащими на $r$ прямых $L_{i_{1}}, \ldots, L_{i_{r}}$, принадлежаших конфигурации.

$\mathrm{B}$ дальнейшем назовем $r$ элементов из $(\mathbb{Z} / m \mathbb{Z})^{k}$ линейно независимыми над $\mathbb{Z} / m \mathbb{Z}$, если они порождают в $(\mathbb{Z} / m \mathbb{Z})^{k}$ подгруппу, изоморфную $(\mathbb{Z} / m \mathbb{Z})^{r}$ (и потому допускающую $(\mathbb{Z} / m \mathbb{Z})^{k-r}$ в качестве дополнения).

ЛЕмма 1.1. Пусть $p$ - двукратная точка конфигурации $L$, и пусть $\varphi\left(\lambda_{i_{1}}\right)$ $u \varphi\left(\lambda_{i_{2}}\right)$ линейно независимь над $\mathbb{Z} / m \mathbb{Z}$ в $(\mathbb{Z} / m \mathbb{Z})^{k}$. Тогда поверхность $Y$ неособа в каждой точке из $f^{-1}(p)$. 
ДокАЗАТЕЛЬСТво. Пусть $p=L_{i_{1}} \cap L_{i_{2}}$. Выберем маленькую окрестность $U$ точки $p$ в $\mathbb{P}^{2}$, изоморфную диску, и локальные координаты $y_{1}, y_{2}$ в $U$ так, что $y_{j}=0$ является уравнением прямой $L_{i_{j}}$. Тогда $H_{1}\left(U \backslash\left(L_{i_{1}} \cup L_{i_{2}}\right), \mathbb{Z}\right) \simeq \mathbb{Z} \oplus \mathbb{Z}$. В каждой точке $q \in g^{-1}(p)$ росток $V \rightarrow U$ накрытия $Y \rightarrow \mathbb{P}^{2}$ является $G^{\prime}$-накрытием, где $G^{\prime}$ есть образ группы $H_{1}\left(U \backslash\left(L_{i_{1}} \cup L_{i_{2}}\right), \mathbb{Z}\right)$ при композиции $\varphi \circ i_{*}$ эпиморфизма $\varphi$ с гомоморфизмом включения $i_{*}: H_{1}\left(U \backslash\left(L_{i_{1}} \cup L_{i_{2}}\right), \mathbb{Z}\right) \rightarrow H_{1}\left(\mathbb{P}^{2} \backslash L, \mathbb{Z}\right)$. Более того, это $G^{\prime}$-накрытие определяется гомоморфизмом $\varphi \circ i_{*}$. Отождествляя $\varphi\left(\lambda_{i_{1}}\right), \varphi\left(\lambda_{i_{2}}\right)$ со стандартными образующими групшы $(\mathbb{Z} / m \mathbb{Z})^{2}$, получим изоморфизм между $V \rightarrow U$ и накрытием, заданным уравнениями $z_{1}^{m}=y_{1}, z_{2}^{m}=y_{2}$. Следовательно, $V$ является неособой поверхностью.

В приведенных далее примерах, чтобы разрешить особые точки на $Y$, лежащие над $r$-кратными точками конфигурации $L$ с $r \geqslant 3$, произведем раздутие всех $r$-кратных точек.

Пусть $\sigma: \widetilde{\mathbb{P}^{2}} \rightarrow \mathbb{P}^{2}$ - это раздутие, $L_{i}^{\prime}$ - собственный прообраз прямой $L_{i}, E_{p}-$ исключительная кривая над $r$-кратной точкой $p$ и $\varepsilon_{p} \in H_{1}\left(\widetilde{\mathbb{P}^{2}} \backslash \sigma^{-1}(L), \mathbb{Z}\right)=$ $H_{1}\left(\mathbb{P}^{2} \backslash L, \mathbb{Z}\right)$ - простая петля вокруг $E_{p}$.

В результате отождествления $H_{1}\left(\widetilde{\mathbb{P}^{2}} \backslash \sigma^{-1}(L), \mathbb{Z}\right)$ с $H_{1}\left(\mathbb{P}^{2} \backslash L, \mathbb{Z}\right)$ эпиморфизм $\varphi$ преобразуется в эпиморфизм $\varphi: H_{1}\left(\widetilde{\mathbb{P}^{2}} \backslash \sigma^{-1}(L), \mathbb{Z}\right) \rightarrow(\mathbb{Z} / m \mathbb{Z})^{k}$. Рассмотрим связанное с ним накрытие Галуа $f: X \rightarrow \widetilde{\mathbb{P}^{2}}$.

Лемма 1.2. Пусть $p=L_{i_{1}} \cap \cdots \cap L_{i_{r}}-r$-кратная точка в L. Tогда $\varepsilon_{p}=\lambda_{i_{1}}+\cdots+\lambda_{i_{r}}$.

ЛЕмма 1.3. Если для каждой $r$-кратной точки $p=L_{i_{1}} \cap \cdots \cap L_{i_{r}}$ в $L$, $r \geqslant 3$, и для каждого $j, \quad 1 \leqslant j \leqslant r$, әлементь $\varphi\left(\varepsilon_{p}\right)$ и $\varphi\left(\lambda_{i_{j}}\right)$ являются линейно независимыми над $\mathbb{Z} / m \mathbb{Z}$ в $(\mathbb{Z} / m \mathbb{Z})^{k}$, то $X$ неособо.

Доказательства этих лемм ввиду их простоты будут опущены (чтобы установить соотношение, данное в лемме 1.2 , достаточно рассмотреть обший пучок прямых; лемма 1.3 следует из леммы 1.1).

В качестве следствия получаем, что построенная поверхность $X$ является разрешением особенностей поверхности $Y$. Действительно, накрытие $f$ может быть включено в коммутативную диаграмму

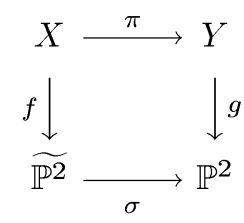

в которой $\pi$ является регулярным отображением (ясно, что оно непрерьвно, и поэтому регулярность следует, например, из регулярности на $\left.X \backslash f^{-1}\left(\sigma^{-1}(L)\right)\right)$.

\section{§2. $(\mathbb{Z} / 5 \mathbb{Z})^{2}$-накрытия Галуа, разветвленные над конфигурацией прямых, двойственных точкам перегиба гладкой кубики}

В этом параграфе мы используем обозначения из $\S 1$.

Пусть $L=L_{1} \cup \cdots \cup L_{9}-$ конфигурация девяти прямых в $\mathbb{P}^{2}$, двойственная девяти точкам перегиба гладкой кубики $C$, лежашей в двойственной плоскости. Пусть $t_{r}$, 
$r \geqslant 2,-$ число $r$-кратных точек конфигурации $L$, т.е. число точек, лежащих ровно на $r$ прямых, принадлежаших конфигурации. Как известно (и легко проверить, используя групповой закон на кубике), для этой конфигурации $t_{3}=12, t_{r}=0$, если $r \neq 3$, и ровно четыре особые точки конфигурации $L$ лежат на каждой прямой $L_{i}$, $1 \leqslant i \leqslant 9$. (Отметим, что конфигурация прямых, двойственная точкам перегиба гладкой кубики, является жесткой, т.е. любая такая конфигурация может быть переведена в другую с помошью линейного преобразования проективной плоскости.)

Если кубика $C$ задана уравнением $x_{1}^{3}+x_{2}^{3}+x_{3}^{3}=0$, то прямые $L_{1}, \ldots, L_{9}$ задаются уравнениями

$$
\begin{array}{lll}
L_{1}=\left\{x_{1}-x_{3}=0\right\}, & L_{2}=\left\{x_{1}-\mu^{2} x_{3}=0\right\}, & L_{3}=\left\{x_{1}+\mu x_{3}=0\right\}, \\
L_{4}=\left\{x_{2}-\mu^{2} x_{3}=0\right\}, & L_{5}=\left\{x_{2}-x_{3}=0\right\}, & L_{6}=\left\{x_{2}+\mu x_{3}=0\right\}, \\
L_{7}=\left\{x_{1}+\mu x_{2}=0\right\}, & L_{8}=\left\{x_{1}-\mu^{2} x_{2}=0\right\}, & L_{9}=\left\{x_{1}-x_{2}=0\right\},
\end{array}
$$

где $\mu=e^{\pi i / 3}$.

Пересечение трех различных прямых $L_{i}, L_{j}, L_{k}$ не пусто тогда и только тогда, когда $(i, j, k) \in T$, где

$$
\begin{aligned}
& T=\{(1,2,3),(4,5,6),(7,8,9),(1,4,7),(2,5,8),(3,6,9), \\
&(1,5,9),(3,5,7),(1,6,8),(3,4,8),(2,4,9),(2,6,7)\} .
\end{aligned}
$$

Обозначим через $p_{i, j, k},(i, j, k) \in T$, точки пересечения прямых $L_{i}, L_{j}, L_{k}$.

Рассмотрим накрытие Галуа $g: Y \rightarrow \mathbb{P}^{2}$ с группой Галуа $G \simeq(\mathbb{Z} / 5 \mathbb{Z})^{2}$, разветвленное вдоль $L$ и заданное эпиморфизмом $\varphi: H_{1}\left(\mathbb{P}^{2} \backslash L, \mathbb{Z}\right) \rightarrow G$.

Обозначим через $\sigma: \widetilde{\mathbb{P}^{2}} \rightarrow \mathbb{P}^{2}$ раздутие с центрами во всех 3 -кратных точках $p_{i, j, k},(i, j, k) \in T$, через $E_{i, j, k}$ - исключительный дивизор над $p_{i, j, k}$ и через $L_{i}^{\prime}-$ собственный прообраз прямой $L_{i}$. Пусть элемент $\varepsilon_{i, j, k} \in H_{1}\left(\widetilde{\mathbb{P}^{2}} \backslash \sigma^{-1}(L), \mathbb{Z}\right) \simeq$ $H_{1}\left(\mathbb{P}^{2} \backslash L, \mathbb{Z}\right)$ соответствует простому обходу вокруг $E_{i, j, k}$.

Эпиморфизм $\varphi: H_{1}\left(\widetilde{\mathbb{P}^{2}} \backslash \sigma^{-1}(L), \mathbb{Z}\right) \rightarrow(\mathbb{Z} / 5 \mathbb{Z})^{2}$ определяет накрытие Галуа $f: X \rightarrow \widetilde{\mathbb{P}^{2}}$. Положим $C_{i}=f^{-1}\left(L_{i}^{\prime}\right)$ и $D_{i, j, k}=f^{-1}\left(E_{i, j, k}\right)$.

В дальнейшем мы предполагаем, что эпиморфизм $\varphi: H_{1}\left(\widetilde{\mathbb{P}^{2}} \backslash \sigma^{-1}(L), \mathbb{Z}\right) \rightarrow$ $(\mathbb{Z} / 5 \mathbb{Z})^{2}$ удовлетворяет следующему условию:

$(\mathrm{S})$ әлементы $\varphi\left(\varepsilon_{i_{1}, i_{2}, i_{3}}\right)$ и $\varphi\left(\lambda_{i_{j}}\right), j=1,2,3$, линейно независимы над $\mathbb{Z} / 5 \mathbb{Z}$ для каждой тройки $\left(i_{1}, i_{2}, i_{3}\right) \in T$.

Данное условие означает, что $f$ разветвлен в каждой кривой $C_{i}$ и каждой кривой $D_{i, j, k}$ с индексом ветвления, равным 5. Более того, согласно лемме 1.3 при этом условии поверхность $X$ является неособой.

ЛЕмма 2.1. При сделанных предположениях:

(i) $C_{i}^{2}=-3$ для каждого $i=1, \ldots, 9$;

(ii) $D_{i_{1}, i_{2}, i_{3}}^{2}=-1$ для каждого набора $\left(i_{1}, i_{2}, i_{3}\right) \in T$;

(iii) $K_{X}^{2}=333$, әде $K_{X}-$ канонический класс поверхности $X$;

(iv) геометрические роды кривых $C_{i}, \quad 1 \leqslant i \leqslant 9, u D_{i_{1}, i_{2}, i_{3}}, \quad\left(i_{1}, i_{2}, i_{3}\right) \in T$, равны $g\left(C_{i}\right)=4$ и $g\left(D_{i_{1}, i_{2}, i_{3}}\right)=2$. 
ДокАЗАТЕльСтво. (i) На каждой $L_{i}$ лежит ровно четыре тройных точки конфигурации $L$. Следовательно, $\left(L_{i}^{\prime}, L_{i}^{\prime}\right)=-3$. С другой стороны,

$$
\operatorname{deg} f \cdot\left(L_{i}^{\prime}, L_{i}^{\prime}\right)=\left(f^{*}\left(L_{i}^{\prime}\right), f^{*}\left(L_{i}^{\prime}\right)\right)=\left(5 C_{i}, 5 C_{i}\right)=25 C_{i}^{2} .
$$

Поэтому $C_{i}^{2}=-3$.

Доказательство утверждения (ii) аналогично доказательству (i).

(iii) 3-канонический класс поверхности $\widetilde{\mathbb{P}^{2}}$ равен $3 K_{\widetilde{\mathbb{P}^{2}}}=-\sum L_{i}$.

По формуле для канонического класса прообраза имеем

$$
K_{X}=f^{*}\left(K_{\widetilde{\mathbb{P}^{2}}}\right)+4\left(\sum C_{i}+\sum D_{i_{1}, i_{2}, i_{3}}\right)
$$

и поэтому

$$
3 K_{X}=7 \sum C_{i}+12 \sum D_{i_{1}, i_{2}, i_{3}} .
$$

Таким образом, получаем

$$
\begin{aligned}
9 \cdot K_{X}^{2} & =49 \sum C_{i}^{2}+144 \sum D_{i_{1}, i_{2}, i_{3}}^{2}+168 \sum\left(C_{i}, D_{i_{1}, i_{2}, i_{3}}\right) \\
& =49 \cdot(-3) \cdot 9+144 \cdot(-1) \cdot 12+168 \cdot 4 \cdot 9 .
\end{aligned}
$$

Следовательно, $K_{X}^{2}=333$.

Из (2.1) следует, что

$$
\left(C_{i}, K_{X}\right)=9, \quad\left(D_{i_{1}, i_{2}, i_{3}}, K_{X}\right)=3,
$$

и утверждение (iv) следует из формулы присоединения.

ЛЕмма 2.2. Поверхность $X$ является поверхностью общего типа с обильным каноническим классом.

ДокАЗАТЕЛЬСтво. Согласно критерию Накаи-Мойшезона достаточно показать, что $\left(K_{X}, C\right)>0$ для любой алгебраической кривой $C \subset X$. Из $(2.1)$ и $(2.2)$ следует, что $\left(K_{X}, C\right) \geqslant 0$ для любой кривой $C$. Предположим, что сушествует неприводимая кривая $C$ такая, что $\left(K_{X}, C\right)=0$. Тогда пересечение кривой $C$ и эффективного дивизора $3 K_{X}=7 \sum C_{i}+12 \sum D_{i_{1}, i_{2}, i_{3}}$ пусто. Следовательно, кривая $\sigma(f(C))$ не пересекается ни с одной из прямых $L_{i}, i=1, \ldots, 9$, что невозможно.

Лемма 2.3. Эйлерова характеристика е $(X)$ поверхности $X$ равна $111, u$, в частности, выполнено соотночение $K_{X}^{2}=3 e(X)$.

ДокАЗАТЕльСтво. Так как $e\left(\widetilde{\mathbb{P}^{2}}\right)=15$ и $e\left(L_{i}\right)=e\left(E_{i_{1}, i_{2}, i_{3}}\right)=2$, то из аддитивности эйлеровой характеристики следует, что

$$
\begin{aligned}
e(X)= & 25 e\left(\widetilde{\mathbb{P}^{2}} \backslash\left(\cup C_{i} \cup D_{i_{1}, i_{2}, i_{3}}\right)\right)+5 \sum e\left(C_{i} \backslash \cup D_{i_{1}, i_{2}, i_{3}}\right) \\
& +5 \sum e\left(D_{i_{1}, i_{2}, i_{3}} \backslash \cup C_{i}\right)+\sum\left(C_{i}, D_{i_{1}, i_{2}, i_{3}}\right) \\
= & 25(15-9 \cdot 2-12 \cdot 2+9 \cdot 4)+5 \cdot 9(2-4)+5 \cdot 12(2-3)+9 \cdot 4=111 .
\end{aligned}
$$

Соотношение $K_{X}^{2}=3 e(X)$ следует из леммы 2.1 , (iii).

СлЕДСТвИЕ 2.1. Поверхность $Х$ является сильно жесткой (т.е. поверхностью, иве пространство модулей сводится либо $к X$ и $\bar{X}$, либо просто $к$ $X$, где $\bar{X}$ означает комплексно-сопряженную поверхность). 


\section{§ 3. Автоморфизмы накрытий}

Пусть $f: X \rightarrow \widetilde{\mathbb{P}^{2}}$ является $(\mathbb{Z} / 5 \mathbb{Z})^{2}$-накрытием Галуа, рассмотренным в $\S 2$. Обозначим через $\mathrm{Kl}$ группу голоморфных и антиголоморфных диффеоморфизмов $X \rightarrow X$. Ясно, что если $\mathrm{Kl}$ содержит по крайней мере один антиголоморфный элемент, то голоморфные элементы в Kl составляют подгруппу Aut индекса 2. Другими словами, мы имеем короткую точную последовательность $1 \rightarrow \mathrm{Aut} \rightarrow \mathrm{Kl} \rightarrow$ $H \rightarrow 1$, где $H=\mathbb{Z} / 2$ либо $H=0$. Обозначим через $\mathrm{kl}: \mathrm{Kl} \rightarrow H$ соответствуюший гомоморфизм в этой последовательности. Напомним, что согласно определению вещественная структура - это антиголоморфная инволющия, и отметим также, что группа $H$ может быть нетривиальна и для многообразий, не обладаюших вещественной структурой.

Групша $\mathrm{Kl}$ действует наиболее естественно на $X \times \bar{X}, X \sqcup \bar{X}(\bar{X}$ - поверхность, комплексно-сопряженная поверхности $X)$ и на связанных с ними группах, таких как Div, Pic и $H^{*}$, а также на $\mathbb{C}(X \times \bar{X})$ и $\mathbb{C}(X \sqcup \bar{X})$ (последнее множество функций не является полем, так как $X \sqcup \bar{X}$ - приводимая поверхность). Существует несколько путей, как с помощью этих действий определить действие групшы $\mathrm{Kl}$, продолжающее действие Aut на $\mathbb{C}(X), \operatorname{Div}(X), \operatorname{Pic}(X)$ и $H^{*}(X)$. Выбираем тот, которьй наиболее приспособлен для данного исследования. Кроме того, именно он является более традиционным в алгебраической геометрии.

Чтобы продолжить действие группы $\operatorname{Aut}(X)$ на $\mathbb{C}(X)$ до действия $\mathrm{Kl}(X)$, свяжем с каждым антиголоморфным диффеоморфизмом $h \mathbb{C}$-антилинейное отображение $h^{!}: \mathbb{C}(X) \rightarrow \mathbb{C}(X)$, заданное формулой $h^{!}(f)(x)=\overline{f(h(x))}, f \in \mathbb{C}(X)$, $x \in X(\mathbb{C})$. Это действие $h^{!}$может быть продолжено на голоморфные дифференциальные формы, если положить

$$
h^{!}(d f)=d h^{!}(f) .
$$

Антиголоморфный диффеоморфизм $h$ определяет действие на $\operatorname{Div}(X)$ : если дивизор $C \in \operatorname{Div}(X)$ задан локальными уравнениями $\left(U_{\alpha}, f_{\alpha}\right)$, то $h(C)$ задается уравнениями $\left(h^{-1}\left(U_{\alpha}\right), \overline{f_{\alpha} \circ h}\right)$. Имеем

$$
h^{-1}(\operatorname{div} f)=\operatorname{div} h^{!}(f), \quad f \in \mathbb{C}(X) .
$$

В соответствии с (3.1) $h: \operatorname{Div}(X) \rightarrow \operatorname{Div}(X)$ индуцирует действие $h^{!}: \operatorname{Pic}(X) \rightarrow$ $\operatorname{Pic}(X)$. Ясно, что канонический класс $K_{X} \in \operatorname{Pic}(X)$ инвариантен при действии $h^{!}$для любого $h \in \mathrm{Kl}$ (здесь и далее мы пологаем $h^{!}=h^{*}$ для $h \in \operatorname{Aut} X$ ). Индекс пересечения дивизоров также сохраняется при действии любого элемента $h \in \mathrm{Kl}$ (заметим, что для $h \in \mathrm{Kl} \backslash$ Aut действие $h^{!}$на группе Нерона-Севери, рассматриваемой как подгруппа в $H^{2}(X, \mathbb{Z})$, совпадает с ограничением действия $-h^{*}: H^{2}(X) \rightarrow H^{2}(X)$ на эту подгруппу, где $h^{*}$ - стандартное действие диффеоморфизма $h$ на когомологиях).

Мы говорим, что $h \in \mathrm{Kl}(X)$ поднят с $\widetilde{\mathbb{P}^{2}}$, если сушествует такой диффеоморфизм $\widetilde{h} \in \mathrm{Kl}\left(\widetilde{\mathbb{P}^{2}}\right)$, что является коммутативной следующая диаграмма:

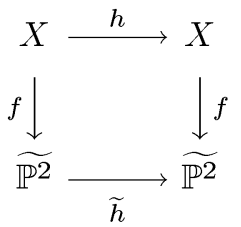


ПРЕДЛОЖЕНИЕ 3.1. Каждый диффеоморфизм $h \in \mathrm{Kl}(X)$ поднят $c \widetilde{\mathbb{P}^{2}} . \quad B$ частности, если $X$ обладает вещественной структурой, то для выбранной соответствующим образом вещественной структуры на $\widetilde{\mathbb{P}^{2}}$ накрытие $f$ определено над $\mathbb{R}$.

Лемма 3.1. Пусть $h \in \mathrm{Kl}(X)$. Тогда $h$ оставляет неподвижными множества $\cup C_{i} u \cup D_{i_{1}, i_{2}, i_{3}}$.

ДокАЗАТЕЛЬСТво. Предположим, что $h\left(C_{i_{0}}\right) \not \subset \cup C_{i}$ для некоторого $i_{0}$. Тогда

$$
\left(h\left(C_{i_{0}}\right), \sum C_{i}\right)=a, \quad a \geqslant 0 .
$$

Так как $g\left(C_{i_{0}}\right) \neq g\left(D_{i_{1}, i_{2}, i_{3}}\right)$, то $h\left(C_{i_{0}}\right) \neq D_{i_{1}, i_{2}, i_{3}}$. Поэтому

$$
\left(h\left(C_{i_{0}}\right), \sum D_{i_{1}, i_{2}, i_{3}}\right)=b, \quad b \geqslant 0 .
$$

Так как $h^{!}\left(K_{X}\right)=K_{X}$, то из леммы 2.1 и формулы присоединения следует, что

$$
\left(h\left(C_{i_{0}}\right), K_{X}\right)=\left(C_{i_{0}}, K_{X}\right)=9 .
$$

Таким образом, ввиду (2.1) и (2.2) должно вьполняться равенство

$$
7 a+12 b=27
$$

для некоторых неотрицательных целых чисел $a$ и $b$, что невозможно.

Доказательство того, что $h\left(D_{i_{1}, i_{2}, i_{3}}\right) \subset \bigcup_{\left(i_{1}, i_{2}, i_{3}\right) \in T} D_{i_{1}, i_{2}, i_{3}}$ для каждой тройки $\left(i_{1}, i_{2}, i_{3}\right) \in T$, аналогично доказательству, приведенному выше.

ДОКАЗАТЕЛЬСТВО ПРЕДЛОЖЕНИЯ 3.1. Второе утверЖдение является непосредственным следствием первого. Чтобы доказать первое утверждение, достаточно показать, что $h$ действует на слоях накрытия $f$, т.е. что для почти всех $p \in \widetilde{\mathbb{P}^{2}}$ можно найти такие $q \in \widetilde{\mathbb{P}^{2}}$, что $h\left(f^{-1}(p)\right)=f^{-1}(q)$.

Зафиксируем точку $p_{i_{0}, j_{0}, k_{0}} \in \mathbb{P}^{2}$. Так как $C_{i_{0}}$ и $C_{j_{0}}$ пересекают $D_{i_{0}, j_{0}, k_{0}}$, то $h\left(C_{i_{0}}\right)$ и $h\left(C_{j_{0}}\right)$ пересекают $h\left(D_{i_{0}, j_{0}, k_{0}}\right)$. Кривая $C_{i_{0}}$ (соответственно, $\left.C_{j_{0}}\right)$ пересекает три других кривых $D_{i_{r}, j_{r}, k_{r}}, r=1,2,3$ (соответственно, $D_{i_{r}, j_{r}, k_{r}}^{\prime}, r=$ $1,2,3)$, отличных от $D_{i_{0}, j_{0}, k_{0}}$. Таким образом, $h\left(C_{i_{0}}\right)$ (соответственно, $\left.h\left(C_{j_{0}}\right)\right)$ пересекает каждую из кривых $h\left(D_{i_{r}, j_{r}, k_{r}}\right), r=1,2,3$ (соответственно, $h\left(D_{i_{r}, j_{r}, k_{r}}^{\prime}\right)$, $r=1,2,3)$.

По лемме $3.1 h\left(C_{i_{0}}\right)=C_{i}$ и $h\left(C_{j_{0}}\right)=C_{j}$ для некоторых $i$ и $j$. Имеем

$$
\operatorname{div} f^{*}\left(l_{i_{0}} l_{j_{0}}^{-1}\right)=5\left(C_{i_{0}}+\sum_{r=1}^{3} D_{i_{r}, j_{r}, k_{r}}\right)-5\left(C_{j_{0}}+\sum_{r=1}^{3} D_{i_{r}, j_{r}, k_{r}}^{\prime}\right)
$$

и

$$
\operatorname{div} f^{*}\left(l_{i} l_{j}^{-1}\right)=5\left(h\left(C_{i_{0}}\right)+\sum_{r=1}^{3} h\left(D_{i_{r}, j_{r}, k_{r}}\right)\right)-5\left(h\left(C_{j_{0}}\right)+\sum_{r=1}^{3} h\left(D_{i_{r}, j_{r}, k_{r}}^{\prime}\right)\right) .
$$


Следовательно, сушествует константа $k_{i_{0}, j_{0}}$ такая, что

$$
h^{!}\left(f^{*}\left(l_{i} l_{j}^{-1}\right)\right)=k_{i_{0}, j_{0}} f^{*}\left(l_{i_{0}} l_{j_{0}}^{-1}\right) .
$$

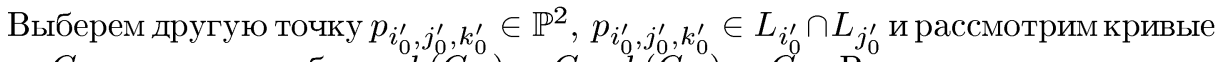
$C_{i_{0}^{\prime}}$ и $C_{j_{0}^{\prime}}$, а также их образы $h\left(C_{i_{0}^{\prime}}\right)=C_{i^{\prime}}, h\left(C_{j_{0}^{\prime}}\right)=C_{j^{\prime}}$. Рассуждения, аналогичные приведенным выше, показывают, что существует константа $k_{i_{0}^{\prime}, j_{0}^{\prime}}$ такая, что

$$
h^{!}\left(f^{*}\left(l_{i^{\prime}} l_{j^{\prime}}^{-1}\right)\right)=k_{i_{0}^{\prime}, j_{0}^{\prime}} f^{*}\left(l_{i_{0}^{\prime}} l_{j_{0}^{\prime}}^{-1}\right)
$$

Так как каждая точка $p \in \widetilde{\mathbb{P}^{2}} \backslash \cup D_{i_{1}, i_{2}, i_{3}}$ может быть задана как пересечение слоев двух линейных рациональных функций $l_{i_{0}} l_{j_{0}}^{-1}$ и $l_{i_{0}^{\prime}} l_{j_{0}^{\prime}}^{-1}$, то из (3.2) и (3.3) следует, что для любой точки $p \in \widetilde{\mathbb{P}^{2}} \backslash \cup D_{i_{1}, i_{2}, i_{3}}$ найдется точка $q \in \widetilde{\mathbb{P}^{2}}$ такая, что $h\left(f^{-1}(p)\right)=f^{-1}(q)$.

\section{$\S 4$. Три примера}

ПРИмеР 4.1. Невещественная жесткая поверхность. Пусть $L=L_{1} \cup$ $\cdots \cup L_{9}-$ конфигурация девяти прямых в $\mathbb{P}^{2}$, двойственная девяти точкам перегиба гладкой кубики $C$, лежащей в двойственной плоскости (см. $\S 2$ ), и пусть $f: X_{1} \rightarrow \widetilde{\mathbb{P}^{2}}$ - накрытие Галуа, ассоциированное с эпиморфизмом $\varphi_{1}: H_{1}\left(\mathbb{P}^{2} \backslash\right.$ $L, \mathbb{Z}) \rightarrow(\mathbb{Z} / 5 \mathbb{Z})^{2}$, заданным на образуюших формулами

$$
\begin{array}{lll}
\varphi_{1}\left(\lambda_{1}\right)=(1,1), & \varphi_{1}\left(\lambda_{2}\right)=(1,0), & \varphi_{1}\left(\lambda_{3}\right)=(1,1), \\
\varphi_{1}\left(\lambda_{4}\right)=(3,3), & \varphi_{1}\left(\lambda_{5}\right)=(3,0), & \varphi_{1}\left(\lambda_{6}\right)=(0,1), \\
\varphi_{1}\left(\lambda_{7}\right)=(0,1), & \varphi_{1}\left(\lambda_{8}\right)=(0,2), & \varphi_{1}\left(\lambda_{9}\right)=(1,1)
\end{array}
$$

(см. $\S 1)$. Отметим, что $\sum \varphi_{1}\left(\lambda_{i}\right)=0 \bmod 5$.

ПРЕДЛОЖЕНИЕ 4.1. Поверхность $X_{1}$ является гладкой и сильно жесткой. Группа $\mathrm{Kl}\left(X_{1}\right)$ совпадает с группой накрывающих преобразований $G=\mathbb{Z} / 5 \times$ $\mathbb{Z} / 5$. В частности, на поверхности $X_{1}$ нет не только никакой вещественной структуры, но даже никаких антиголоморфных диффеоморфизмов.

ДоКАЗАТЕЛЬСТвО. Поверхность $X_{1}$ является гладкой ввиду леммы 1.3. Согласно леммам 2.1 и 2.3 имеем $K_{X_{1}}^{2}=333$ и $e\left(X_{1}\right)=111$, и утверждение о жесткости следует из следствия 2.1.

Рассмотрим произвольньй элемент $c \in \mathrm{Kl}\left(X_{1}\right)$. По предложению $3.1 c$ поднят с $\widetilde{\mathbb{P}^{2}}$, т.е. сушествует диффеоморфизм $\tilde{c} \in \operatorname{Kl}\left(\widetilde{\mathbb{P}^{2}}\right)$ такой, что $f \circ c=\tilde{c} \circ f$.

Как и в $\S 1$ рассмотрим аффинные координаты $x_{1}, x_{2}$ в $\mathbb{C}^{2}=\mathbb{P}^{2} \backslash L_{\infty}$ и линейные уравнения $l_{i}\left(x_{1}, x_{2}\right)=0$ прямых $L_{i} \cap \mathbb{C}^{2}$. Тогда поле рациональных функций $\mathbb{C}\left(X_{1}\right)$ на $X_{1}$ может быть отождествлено с подполем

$$
K_{\varphi_{1}}=\mathbb{C}\left(x_{1}, x_{2}, w_{1}, w_{2}\right)
$$


поля $K_{u(5)}$, где $w_{1}^{5}=l_{1} l_{2} l_{3} l_{4}^{3} l_{5}^{3} l_{9}$ и $w_{2}^{5}=l_{1} l_{3} l_{4}^{3} l_{6} l_{7} l_{8}^{2} l_{9}$, так, что

$$
K_{\varphi_{1}}=\bigoplus_{a \in \operatorname{pr} A_{1}} \mathbb{C}\left(x_{1}, x_{2}\right) z^{a}
$$

является подпространством векторного пространства

$$
K_{u(m)}=\bigoplus_{a \in \operatorname{pr} A} \mathbb{C}\left(x_{1}, x_{2}\right) z^{a}
$$

над $\mathbb{C}\left(x_{1}, x_{2}\right)$, где

$$
A=\left\{\alpha=\left(\alpha_{1}, \ldots, \alpha_{9}\right) \in \mathbb{Z}^{9} \mid 0 \leqslant \alpha_{i} \leqslant 4 \text { и } \sum \alpha_{i}=0 \bmod 5\right\},
$$

$\operatorname{pr}: A \mapsto \bar{A} \simeq(\mathbb{Z} / 5 \mathbb{Z})^{8}-$ проекция, заданная $\operatorname{pr}(\alpha)=\left(\alpha_{1}, \ldots, \alpha_{8}\right)$ для $\alpha=$ $\left(\alpha_{1}, \ldots, \alpha_{9}\right)$, и $A_{1} \subset A$ состоит из $0=(0,0,0,0,0,0,0,0,0)$ и из элементов

$(1,1,1,3,3,0,0,0,1), \quad(2,2,2,1,1,0,0,0,2), \quad(3,3,3,4,4,0,0,0,3), \quad(4,4,4,2,2,0,0,0,4)$, $(1,0,1,3,0,1,1,2,1), \quad(2,0,2,1,0,2,2,4,2), \quad(3,0,3,4,0,3,3,1,3), \quad(4,0,4,2,0,4,4,3,4)$, $(2,1,2,1,3,1,1,2,2), \quad(4,2,4,2,1,2,2,4,4), \quad(1,3,1,3,4,3,3,1,1), \quad(3,4,3,4,2,4,4,3,3)$, $(3,1,3,4,3,2,2,4,3), \quad(1,2,1,3,1,4,4,3,1), \quad(4,3,4,2,4,1,1,2,4), \quad(2,4,2,1,2,3,3,1,2)$, $(4,1,4,2,3,3,3,1,4), \quad(3,2,3,4,1,1,1,2,3), \quad(2,3,2,1,4,4,4,3,2), \quad(1,4,1,3,2,2,2,4,1)$, $(0,1,0,0,3,4,4,3,0), \quad(0,2,0,0,1,3,3,1,0), \quad(0,3,0,0,4,2,2,4,0), \quad(0,4,0,0,2,1,1,2,0)$.

Диффеоморфизм $c$ индуцирует действие $c^{!}$на $\mathbb{C}\left(X_{1}\right)$ такое, что ограничение $c^{!}$ на подполе $\mathbb{C}\left(\widetilde{\mathbb{P}}^{2}\right)=\mathbb{C}\left(\mathbb{P}^{2}\right)$ совпадает с $\tilde{c}^{!}($см. $\S 3)$. По лемме 3.1 множества $\cup C_{i}$ и $\cup D_{i_{1}, i_{2}, i_{3}}$ инвариантны при действии $c$. Следовательно, множество $\cup L_{i}$ является инвариантным при действии $\tilde{c}$. Таким образом, $c^{!}$действует на множестве одномерных подпространств $\mathbb{C}\left(x_{1}, x_{2}\right) z^{a}, a \in \operatorname{pr} A_{1}$, поля $K_{\varphi_{1}}$ и поэтому индуцирует действие на $A_{1}$, которое мы также будем обозначать через $c$ !. Для $a \in A_{1}$ обозначим через $r_{i}(a), i \in \mathbb{Z} / 5 \mathbb{Z}$, число координат вектора $a$, равных $i$.

ЛЕмма 4.1. Функиия $r_{i}$ инвариантна при действии $c^{!}$, m.e. $r_{i}(\alpha)=r_{i}(\beta)$ $\partial \Omega я \beta=c^{!}(\alpha)$.

ДокАЗАтельство. Для каждого $j, 1 \leqslant j \leqslant 9$, координата $\alpha_{j}$ вектора $\alpha=$ $\left(\alpha_{1}, \ldots, \alpha_{9}\right) \in A_{1}$ сравнима по модулю 5 с порядком нуля вдоль $C_{j}$ каждой функции из $C\left(x_{1}, x_{2}\right) z^{a}, a=\operatorname{pr} \alpha$. Для доказательства леммы осталось заметить, что ввиду леммы $3.1 c$ переставляет кривые $C_{j}$.

Из леммы 4.1 следует, что действие $c^{!}$на $A_{1}$ определяется некоторой перестановкой $\pi$ чисел $1, \ldots, 9$.

Рассмотрим элементы $\alpha=(1,1,1,3,3,0,0,0,1)$ и $\beta=(1,0,1,3,0,1,1,2,1)$. Легко видеть, что $\alpha$ является единственным элементом в $A_{1}$ с $r_{0}=3, r_{1}=4$, $r_{2}=0, r_{3}=2, r_{4}=0$. Соответственно, $\beta$ является единственным элементом в $A_{1}$ с $r_{0}=2, r_{1}=5, r_{2}=1, r_{3}=1, r_{4}=0$. Поэтому по лемме $4.1 c^{!}(\alpha)=\alpha$ и $c^{!}(\beta)=\beta$. Так как $r_{2}(\beta)=1$ и $r_{3}(\beta)=1$, то $\tilde{c}\left(L_{4}\right)=L_{4}$ и $\tilde{c}\left(L_{8}\right)=L_{8}$. Далее, $r_{3}(\alpha)=2$ влечет $\tilde{c}\left(L_{5}\right)=L_{5}$ и $r_{0}(\beta)=2$ влечет $\tilde{c}\left(L_{2}\right)=L_{2}$. 
Полученные выше свойства инвариантности прямых $L_{2}, L_{4}, L_{5}, L_{8}$ означают, что эти прямые являются инвариантными относительно действия $\tilde{c}$. Следовательно, точки $p_{2,4,9}=L_{2} \cap L_{4}, p_{2,5,8}=L_{5} \cap L_{8}, p_{4,5,6}=L_{4} \cap L_{5}$ и $p_{3,4,8}=L_{4} \cap L_{8}$ являются неподвижными относительно действия $\tilde{c}$.

Так как $r_{0}(a)=2$, то имеются две возможности: либо $\tilde{c}\left(L_{6}\right)=L_{7}$ и $\tilde{c}\left(L_{7}\right)=L_{6}$, либо $\tilde{c}\left(L_{6}\right)=L_{6}$ и $\tilde{c}\left(L_{7}\right)=L_{7}$.

Если $\tilde{c}\left(L_{6}\right)=L_{7}$ и $\tilde{c}\left(L_{7}\right)=L_{6}$, то их точка пересечения $p_{2,6,7}$ является неподвижной точкой. Но это невозможно. Действительно, в этом случае $L_{6}$ проходит через две различные неподвижные точки $p_{2,6,7}$ и $p_{4,5,6}$, и поэтому должна удовлетворять равенству $\tilde{c}\left(L_{6}\right)=L_{6}$.

Если $L_{6}$ и $L_{7}$ являются инвариантными прямыми, то все прямые $L_{i}, 1 \leqslant i \leqslant 9$, должны быть инвариантными. Действительно, так как $L_{5}$ и $L_{7}$ - инвариантные прямые, то их точка пересечения $p_{3,5,7}$ является неподвижной точкой. Поэтому $L_{3}$ является инвариантной прямой, так как $L_{3}$ проходит через две неподвижные точки $p_{3,4,8}$ и $p_{3,5,7}$. Следовательно, точки пересечений: $p_{3,6,9}$ прямых $L_{3}$ и $L_{6}, p_{1,2,3}$ прямых $L_{2}$ и $L_{3}, p_{1,4,7}$ прямых $L_{4}$ и $L_{7}$ и $p_{7,8,9}$ прямых $L_{7}$ и $L_{8}$ - также являются неподвижными точками. Отсюда вытекает, что $L_{1}$ и $L_{9}$, которые проходят соответственно через $p_{1,2,3}, p_{1,4,7}$ и $p_{3,6,9}, p_{7,8,9}$, являются инвариантными прямыми.

Итак, мы доказали, что девять точек перегиба гладкой кубики $C$ являются неподвижными точками при действии на $\mathbb{P}^{2}$, индуцированном $\tilde{c}$. Следовательно, если $\tilde{c} \in \operatorname{Aut}\left(\widetilde{\mathbb{P}^{2}}\right)$, то $\tilde{c}=\operatorname{Id}$ и, следовательно $c$ является накрывающим преобразованием. Если $\tilde{c} \notin \operatorname{Aut}\left(\widetilde{\mathbb{P}^{2}}\right)$, то $\tilde{c}^{2} \in \operatorname{Aut}\left(\widetilde{\mathbb{P}^{2}}\right)$ является тождественным преобразованием, и поэтому $\tilde{c}$ индуцирует вещественную структуру на $\mathbb{P}^{2}$ такую, что все точки перегиба гладкой кубики $C$ являются вещественными относительно этой структуры, но это невозможно.

СлЕДСТВИЕ 4.1. Пространство модулей комплексных структур на подлежащем гладком четырехмерном многообразии состоит из двух различных точек: $X_{1}$ и $\bar{X}_{1}$. В частности, $X_{1}$ и $\bar{X}_{1}$ дают контрпример $\kappa$ "Dif=Def"проблеме $e^{1}$.

ЗАМЕчАНИЕ 4.1. Из предложения 4.1 и сильной жесткости Мостова можно получить (используя неравенство Смита для преобразований простого порядка, а также когомологические рассуждения), что $X_{1}$ не имеет нетривиальных диффеоморфизмов порядков, не равных 5 .

ЗАмЕЧАнИЕ 4.2. Иррегулярность поверхности $X_{1}$ равна нулю. Это следует, например, из [6].

ПрИМер 4.2. Вещественная немаксимальная жсесткая поверхность. Пусть кубика $C$ задана уравнением $x_{1}^{3}+x_{2}^{3}+x_{3}^{3}=0$ и прямые $L_{1}, \ldots, L_{9}$ перенумерованы точно так же, как и в $\S 2$. В частности, при этой нумерации прямые $L_{1}, L_{5}$ и $L_{9}$ являются вешественными. Пусть $f: X_{2} \rightarrow \widetilde{\mathbb{P}^{2}}$ - накрытие Галуа, связанное с эпиморфизмом $\varphi_{2}: H_{1}\left(\mathbb{P}^{2} \backslash L, \mathbb{Z}\right) \rightarrow(\mathbb{Z} / m \mathbb{Z})^{2}$, заданным формулами $\varphi_{2}\left(\lambda_{i}\right)=\left(a_{i, 1}, a_{i, 2}\right)$,

\footnotetext{
${ }^{1}$ Первые контрпримеры к "Dif=Def"-проблеме были построены Манетти в [9].
} 
где

$$
\begin{array}{lll}
\varphi_{2}\left(\lambda_{1}\right)=(0,1), & \varphi_{2}\left(\lambda_{2}\right)=(1,0), & \varphi_{2}\left(\lambda_{3}\right)=(1,0), \\
\varphi_{2}\left(\lambda_{4}\right)=(0,1), & \varphi_{2}\left(\lambda_{5}\right)=(1,0), & \varphi_{2}\left(\lambda_{6}\right)=(0,1), \\
\varphi_{2}\left(\lambda_{7}\right)=(1,2), & \varphi_{2}\left(\lambda_{8}\right)=(1,2), & \varphi_{2}\left(\lambda_{9}\right)=(0,3) .
\end{array}
$$

ПРЕДЛОЖЕНИЕ 4.2. Поверхность $X_{2}$ является гладкой сильно жсесткой поверхностью. Она мохет быть снабжена вещественной структурой. Эта структура единственна с точностью до сопряжения на әлементы из группь накрывающих преобразований и не является максимальной (последнее означает, что $\left.\sum \operatorname{dim} H_{i}\left(X_{2}(\mathbb{R}) ; \mathbb{Z} / 2 \mathbb{Z}\right)<\sum \operatorname{dim} H_{i}\left(X_{2}(\mathbb{C}) ; \mathbb{Z} / 2 \mathbb{Z}\right)\right)$. Группа $\mathrm{Kl}\left(X_{2}\right)$ является полупрямым произведением группь второго порядка $\mu_{2} \simeq \mathbb{Z} / 2 u$ группь накрьвающих преобразований $G \simeq \mathbb{Z} / 5 \times \mathbb{Z} / 5$. Это $\mathbb{Z} / 2$-расиирение определено соотношениями $s \gamma s^{-1}=\gamma^{-1}, \gamma \in G, s \in \mu_{2}, s \neq 1$.

ДокАЗАТЕЛьСтво. Как и в доказательстве предложения 4.1, $X_{2}$ является гладкой поверхностью ввиду леммы 1.3. Согласно леммам 2.1 и 2.3 мы имеем $K_{X_{2}}^{2}=333$ и $e\left(X_{2}\right)=111$, и утверждение о жесткости следует из следствия 2.1 .

Как и выше, мы отождествляем поле рациональных функций $\mathbb{C}\left(X_{2}\right)$ на $X_{2}$ с подполем $K_{\varphi_{2}}=\mathbb{C}\left(x_{1}, x_{2}, w_{1}, w_{2}\right)$ в $K_{u(5)}$, где $w_{1}^{5}=l_{2} l_{3} l_{5} l_{7} l_{8}$ и $w_{2}^{5}=l_{1} l_{4} l_{6} l_{7}^{2} l_{8}^{2} l_{9}^{3}$. Тогда

$$
K_{\varphi_{2}}=\bigoplus_{a \in \operatorname{pr} A_{2}} \mathbb{C}\left(x_{1}, x_{2}\right) z^{a}
$$

является подпространством векторного пространства

$$
K_{u(m)}=\bigoplus_{a \in \operatorname{pr} A} \mathbb{C}\left(x_{1}, x_{2}\right) z^{a}
$$

над $\mathbb{C}\left(x_{1}, x_{2}\right)$, где $A_{2}$ состоит из $(0,0,0,0,0,0,0,0,0)$ и из элементов

$(0,1,1,0,1,0,1,1,0), \quad(0,2,2,0,2,0,2,2,0), \quad(0,3,3,0,3,0,3,3,0), \quad(0,4,4,0,4,0,4,4,0)$, $(1,0,0,1,0,1,2,2,3), \quad(2,0,0,2,0,2,4,4,1), \quad(3,0,0,3,0,3,1,1,4), \quad(4,0,0,4,0,4,3,3,2)$, $(1,1,1,1,1,1,3,3,3), \quad(2,2,2,2,2,2,1,1,1), \quad(3,3,3,3,3,3,4,4,4), \quad(4,4,4,4,4,4,2,2,2)$, $(1,2,2,1,2,1,4,4,3), \quad(2,4,4,2,4,2,3,3,1), \quad(3,1,1,3,1,3,2,2,4), \quad(4,3,3,4,3,4,1,1,2)$, $(1,3,3,1,3,1,0,0,3), \quad(2,1,1,2,1,2,0,0,1), \quad(3,4,4,3,4,3,0,0,4), \quad(4,2,2,4,2,4,0,0,2)$, $(1,4,4,1,4,1,1,1,3), \quad(2,3,3,2,3,2,2,2,1), \quad(3,2,2,3,2,3,3,3,4), \quad(4,1,1,4,1,4,4,4,2)$.

Обозначим $\alpha=(0,1,1,0,1,0,1,1,0)$ и $\beta=(1,0,0,1,0,1,2,2,3)$ и рассмотрим произвольный элемент $c \in \mathrm{Kl}\left(X_{2}\right), c \neq \mathrm{Id}$. Те же самые рассуждения, что и в доказательстве предложения 4.1 , показывают, что прямая $L_{9}$ и каждое из объединений $L_{7} \cup L_{8}, L_{1} \cup L_{4} \cup L_{6}$ и $L_{2} \cup L_{3} \cup L_{5}$ являются инвариантными при действии $\tilde{c}$.

Покажем, что равенства $\tilde{c}\left(L_{7}\right)=L_{7}$ и $\tilde{c}\left(L_{8}\right)=L_{8}$ невозможны. Действительно, в противном случае имеем $\tilde{c}\left(p_{1,4,7}\right)=p_{1,4,7}$, так как конфигурация прямых $L_{1} \cup$ $L_{4} \cup L_{6} \cup L_{7} \cup L_{8}$ имеет только две тройные точки $p_{1,4,7}$ и $p_{1,6,8}$. Отсюда следовало бы равенство $\tilde{c}\left(L_{6}\right)=L_{6}$, которое вместе с $\tilde{c}\left(L_{9}\right)=L_{9}$ влекло бы то, что $L_{1}$, а потому и $L_{4}$, и, следовательно, все прямые являются инвариантными относительно действия $\tilde{c}$, что противоречит условию $\tilde{c} \neq \mathrm{Id}$. 
Поэтому мы имеем только одну возможность: $\tilde{c}\left(L_{7}\right)=L_{8}$ и $\tilde{c}\left(L_{8}\right)=L_{7}$. Так как пара тройных точек $\left\{p_{2,5,8}, p_{3,5,7}\right\}$ конфигурации $L_{2} \cup L_{3} \cup L_{5} \cup L_{7} \cup L_{8}$ инвариантна при действии $\tilde{c}$, то прямая $L_{5}$ является инвариантной, а прямые $L_{2}$ и $L_{3}$ переставляются. Те же самые аргументы показывают, что $\tilde{c}\left(L_{1}\right)=L_{1}, \tilde{c}\left(L_{4}\right)=L_{6}$ и $\tilde{c}\left(L_{6}\right)=L_{4}$.

Такое действие $\tilde{c}$ на $L=\cup L_{i}$ совпадает с действием, индуцированным стандартным комплексным сопряжением на $\widetilde{\mathbb{P}^{2}}$ (как это следует из изложенного в $\S 2$ ), и поэтому совпадает с ним. Это действие поднимается и определяет вещественную структуру $s$ на $X_{2}$. Фактически $X_{2}$ может быть рассмотрено как минимальное разрешение особенностей проективного замыкания вешественной поверхности, заданной уравнениями

$$
\begin{aligned}
& w_{1}^{5}=\left(x_{1}^{2}+x_{1}+1\right)\left(x_{2}-1\right)\left(x_{1}^{2}+x_{1} x_{2}+x_{2}^{2}\right), \\
& w_{2}^{5}=\left(x_{1}-1\right)\left(x_{2}^{2}+x_{2}+1\right)\left(x_{1}^{2}+x_{1} x_{2}+x_{2}^{2}\right)^{2}\left(x_{1}-x_{2}\right)^{3} .
\end{aligned}
$$

Эта вешественная поверхность не является максимальной, так как ее вещественная часть гомеоморфна $\mathbb{R} \mathbb{P}^{2}$ с 4 раздутыми точками (легко проверить, что среди раздутых точек $p_{i, j, k} \in T$ имеются только четыре вещественные точки). Так как каждый элемент $c \in \mathrm{Kl}\left(X_{2}\right)$ определяется диффеоморфизмом $\tilde{c}$ однозначно с точностью до композиции с накрьваюшим преобразованием, то группа $\mathrm{Kl}\left(X_{2}\right)$ порождается элементом $s$ и накрываюшими преобразованиями. Соотношения коммутирования $s \gamma=\gamma^{-1} s$ следуют из приведенных вьше уравнений. Эти соотношения влекут то, что каждое $s \gamma$ является вешественной структурой и все эти вешественные структуры эквивалентны друг другу.

ЗАмечАниЕ 4.3. Поверхности в приведенных выше примерах имеют одинаковые значения $K^{2}$ и $e$. Таким образом, они принадлежат одной и той же схеме Гильберта, тем самым они дают пример схемы Гильберта, связные компоненты которой имеют различные свойства относительно сушествования вешественных структур на поверхностях, представляюших эти компоненты. Отметим также, что в противоположность первому примеру во втором примере пространство модулей сводится к одной точке, которая является вешественной (и, более того, соответствует поверхности с вещественной структурой).

ПРИмеР 4.3. Жесткая поверхность с двумя неәквивалентными вещественными структурами. Две вещественные структуры на комплексной поверхности называются әквивалентнылми, если одна из них может быть преобразована в другую с помощью автоморфизма поверхности.

Пусть конфигурация прямых $L=L_{1} \cup \cdots \cup L_{6}$ является полным четырехсторонником. Отметим, что все полные четырехсторонники проективно эквивалентны друг другу. Для данной конфигурации имеем $t_{2}=3, t_{3}=4$ и $t_{r}=0$ для $r \geqslant 4$. Занумеровав подходящим образом прямые, входящие в четырехсторонник, можно считать, что множество 2 -кратных точек состоит из $\left\{L_{1} \cap L_{4}, L_{2} \cap L_{5}, L_{3} \cap L_{6}\right\}$ и множество 3-кратных точек состоит из

$$
\left\{L_{1} \cap L_{2} \cap L_{6}, L_{2} \cap L_{3} \cap L_{4}, L_{1} \cap L_{3} \cap L_{6}, L_{4} \cap L_{5} \cap L_{6}\right\} .
$$


Пусть $f: X_{3} \rightarrow \widetilde{\mathbb{P}^{2}}$ - накрытие Галуа, определяемое эпиморфизмом $\varphi_{3}: H_{1}\left(\mathbb{P}^{2} \backslash\right.$ $L, \mathbb{Z}) \rightarrow(\mathbb{Z} / 5 \mathbb{Z})^{2}$, заданным на образуюших $\lambda_{i}$ группы $H_{1}\left(\mathbb{P}^{2} \backslash L, \mathbb{Z}\right)$ формулами

$$
\begin{array}{lll}
\varphi_{3}\left(\lambda_{1}\right)=(1,0), & \varphi_{3}\left(\lambda_{2}\right)=(1,0), & \varphi_{3}\left(\lambda_{3}\right)=(1,2), \\
\varphi_{3}\left(\lambda_{4}\right)=(0,1), & \varphi_{3}\left(\lambda_{5}\right)=(0,1), & \varphi_{3}\left(\lambda_{6}\right)=(2,1),
\end{array}
$$

где $\widetilde{\mathbb{P}^{2}}$ - раздутие $\mathbb{P}^{2}$ во всех 3 -кратных точках конфигурации $L$. Как и вьше, обозначим $\sigma: \widetilde{\mathbb{P}^{2}} \rightarrow \mathbb{P}^{2}$ это раздутие, $E_{i, j, k}$ - исключительньй дивизор, лежаший над 3 -кратной точкой $p_{i, j, k}$, и $L_{i}^{\prime}-$ собственный прообраз прямой $L_{i}$. Положим $C_{i}=f_{3}^{-1}\left(L_{i}^{\prime}\right)$ и $D_{i, j, k}=f_{3}^{-1}\left(E_{i, j, k}\right)$.

Как и в $\S 1$, рассмотрим аффинные координаты $x_{1}, x_{2}$ в $\mathbb{C}^{2}=\mathbb{P}^{2} \backslash L_{\infty}$, и пусть $l_{i}\left(x_{1}, x_{2}\right)=0$ - линейные уравнения прямых $L_{i} \cap \mathbb{C}^{2}$. Тогда по лемме 1.3 поверхность $X_{3}$ изоморфна минимальному разрешению особенностей проективного замыкания поверхности, заданной уравнениями

$$
\begin{aligned}
w_{1}^{5} & =l_{1} l_{2} l_{3} l_{6}^{2}, \\
w_{2}^{5} & =l_{3}^{2} l_{4} l_{5} l_{6} .
\end{aligned}
$$

Вычисления, аналогичные приведенным в доказательствах лемм 2.1 и 2.2 , показывают, что $X_{3}$ является поверхностью общего типа с $K_{X_{3}}^{2}=45$ и $e\left(X_{3}\right)=15$. Следовательно, $X_{3}$ является сильно жесткой поверхностью.

Лемма 4.2. Пусть $h \in \mathrm{Kl}\left(X_{3}\right)$. Тогда $h$ оставляет на месте множество $\left(\cup C_{i}\right) \cup\left(\cup D_{i_{1}, i_{2}, i_{3}}\right)$.

ДокАЗАТЕЛЬСТВО аналогично доказательству леммы 3.1.

ПРЕДЛОЖЕНИЕ 4.3. Каждый диффеоморфизм $h \in \mathrm{Kl}\left(X_{3}\right)$ является поднятым с $\widetilde{\mathbb{P}^{2}}$. В частности, если $X_{3}$ обладает вещественной структурой, то для подходящим образом выбранной вещественной структуры на $\widetilde{\mathbb{P}^{2}}$ накрытие $f$ определено над $\mathbb{R}$.

ДоКАЗАТЕЛЬСТвО аналогично доказательству предложения 3.1.

ПРЕДЛОЖЕНИЕ 4.4. Поверхность $X_{3}$ обладает двумя неэквивалентными вещественными структурами.

ДокАЗАтельство. Рассмотрим две вешественные структуры на $\mathbb{P}^{2}$. Для первой из них все прямые $L_{i}$ конфигурации $L$ являются вещественными, а для второй прямые $L_{3}, L_{6}$ являются вещественными и $L_{1}, L_{2}$, соответственно $L_{4}$ и $L_{5}$, являются комплексно-сопряженными. Эти две вещественные структуры индуцируют две вещественные структуры на $X_{3}$, так как в обоих случаях многочлены в (4.2) и (4.3) определены над $\mathbb{R}$.

Эти две вешественные структуры на $X_{3}$ не эквивалентны. Действительно, по лемме 4.2 каждый автоморфизм поверхности $X_{3}$ оставляет на месте множество $\left(\cup C_{i}\right) \cup\left(\cup D_{i_{1}, i_{2}, i_{3}}\right)$, в то время как, с одной стороны, все кривые $C_{i}, i=1, \ldots, 6$, и все $D_{i_{1}, i_{2}, i_{3}}$ являются вещественными относительно первой вещественной структуры, но, с другой стороны, только $C_{3}$ и $C_{6}$ (среди кривых $\left.C_{1}, \ldots, C_{6}\right)$ являются вещественными относительно второй вещественной структуры. 


\section{$\S 5$. Невещественность фальшивых проективных поверхностей и несколько замечаний}

5.1. Назовем поверхность основного типа с $p_{g}=q=0$ и $K^{2}=9$ фальшивой проективной плоскостью. Сушествование фальшивых проективных плоскостей доказано Д. Мамфордом [11].

ТЕОРЕма 5.1. Фальшивая проективная плоскость не имеет антиголоморфных диффеоморфизмов.

ДокАЗАТЕЛЬСТво. Пусть $X$ - фальшивая проективная плоскость. Тогда (см. [10], [14]) универсальная накрывающая многообразия $X$ является шаром.

Покажем сначала, что на $X$ нет антиголоморфных инволюций. Предположим противное, т.е. пусть поверхность $X$ снабжена вешественной структурой, и обозначим через $X_{\mathbb{R}}$ множество вешественных точек поверхности $X$. Применяя формулу следа Лефшеца к инволюции, задаюшей вешественную структуру, получаем, что $e\left(X_{\mathbb{R}}\right)=1$. Следовательно, $X_{\mathbb{R}}$ не пусто и содержит по крайней мере одну компоненту, диффеоморфную либо сфере, либо вещественной проективной плоскости. Для того чтобы поднять вещественную структуру на универсальную накрывающую, выберем точку $p$ на такой компоненте и отождествим точки универсальной накрывающей с классами гомотопных путей с началом в точке $p$. Вешественная часть накрытия накрывает (без ветвления) выбранную вешественную компоненту поверхности $X$. С другой стороны, так как универсальная накрываюшая является шаром, ее вешественная часть не имеет компактных компонент.

Из вышесказанного следует, что если существует антиголоморфный диффеоморфизм $h$, то его порядок не может быть равен $2 n$, где $n-$ нечетное число. Действительно, если $n$ - нечетное число, то $h^{n}$ является антиголоморфной инволюцией. Поэтому теорема 5.1 вытекает из следующей леммы.

Лемма 5.1. Группа Aut $X$ не имеет әлементов четного порядка.

ДокАЗАТЕЛЬСтво. Предположим, что сушествует $h \in$ Aut $X$ второго порядка. Одномерные компоненты $C$ множества неподвижных точек автоморфизма $h$ неособы. Согласно относительной пропорциональности Еноки-Хирцебруха [2] выполнено равенство

$$
e(C)=2 C^{2} .
$$

Поэтому $C=\varnothing$, так как иначе $C^{2}>0$, а $e(C)<0$ (эти неравенства следуют, например, из равенства $C=r K$ при некотором положительном $r \in \mathbb{Q}$ ).

Так как $\operatorname{dim} H^{i}(X, \mathbb{C})=1$ для $i=0,2,4$ и $\operatorname{dim} H^{i}(X, \mathbb{C})=0$ для $i=1,3$, то согласно топологической формуле Лефшеца число неподвижных точек автоморфизма $h$ должно равняться 3 для любого нетривиального автоморфизма без одномерных компонент во множестве неподвижных точек. Далее, применяя голоморфную формулу Лефшеца к такому $h$ (второго порядка), имеем

$$
\sum_{i=1}^{3} \frac{1}{\operatorname{det}\left(\operatorname{Id}-D_{i}\right)}=1
$$


где $D_{i}, \quad i=1,2,3,-$ матрицы Якоби автоморфизма $h$ в неподвижных точках. С другой стороны, $\operatorname{det}\left(\mathrm{Id}-D_{i}\right)=4$ в каждой неподвижной точке и, следовательно, формула Лефшеца дает $\frac{3}{4}=1$, т.е. мы получаем противоречие, которое доказывает лемму и завершает доказательство теоремы 5.1.

СЛЕДСТВИЕ 5.1. Для любой фальшивой проективной плоскости пространство модулей комплексных структур на подлежсащем четырехмерном многообразии состоит из двух различных точек: $X$ и $\bar{X}$.

5.2. Аргументы, использованные в доказательстве теоремы 5.1 и исключающие существование антиголоморфных инволюций, можно заменить следующим общим результатом.

ТЕОРЕМА 5.2. Если $X$ является компактной комплексной кәлеровой поверхностью отрицательной секционной кривизнь, то для любой вещественной структуры на $X$ вещественная часть поверхности $X$ не имеет компонент, диффеоморфных сфере, вещественной проективной плоскости, тору или бутылке Клейна.

ДокАЗАТЕльство. Пусть $p: B \rightarrow X-$ универсальное накрытие. Согласно теореме Картана-Адамара пространство $B$ диффеоморфно $\mathbb{R}^{4}$. Каждая связная компонента $M$ прообраза $p^{-1}(F)$ компоненты $F$ вещественной части поверхности $X$ является вещественной компонентой для некоторой вещественной структуры на $B$. Следовательно, по теореме Смита $M$ имеет гомологии, как у точки, и поэтому она диффеоморфна $\mathbb{R}^{2}$. Это исключает сферу и вещественную проективную плоскость в качестве $F$ и влечет инъективность гомоморфизма $\pi_{1}(F) \rightarrow \pi_{1}(X)$. Для доказательства теоремы остается заметить, что $\pi_{1}(X)$, являющаяся фундаментальной группой компактного многообразия отрицательной кривизны, не содержит (см. [13]) подгруппу, изоморфную $\mathbb{Z} \oplus \mathbb{Z}$.

Было бы интересно сравнить это наблюдение с гипотезой Коллара (и теоремой Витербо [8]), согласно которой алгебраическое многообразие размерности $\geqslant 3$ является многообразием общего типа, если одна из компонент его вещественной части (относительно некоторой вещественной структуры) гиперболична.

5.3. Поверхности Мияоки-Яо могут быть источником интересных примеров, относящихся к "неравенству Регсдейла", т.е. примеров вещественных поверхностей $X$ со значениями $\beta_{1}^{\mathbb{R}}=\operatorname{dim} H_{1}\left(X_{\mathbb{R}}, \mathbb{Z} / 2 \mathbb{Z}\right)$, близкими или бо́льшими $h^{1,1}(X)$. (Первые примеры с $\beta_{1}^{\mathbb{R}}>h^{1,1}(X)$ были найдены в начале восьмидесятых годов Итенбергом [7].) Напомним, что вешественная поверхность $X$ называется максимальной (или $M$-поверхностью), если неравенство Смита (см., например, обзор [3])

$$
\sum \beta_{i}^{\mathbb{R}} \leqslant \sum \beta_{i}^{\mathbb{C}}=2+4\left(h^{1,0}+\nu\right)+2 h^{2,0}+h^{1,1}
$$

преврашается в равенство, где $\nu$ - ранг 2 -кручения в $H_{1}(X ; \mathbb{Z})$ и $\beta_{i}^{\mathbb{C}}=\operatorname{dim} H_{i}(X$; $\mathbb{Z} / 2 \mathbb{Z}$ ). 
Согласно формуле Лефшеца

$$
\beta_{0}^{\mathbb{R}}-\beta_{1}^{\mathbb{R}}+\beta_{2}^{\mathbb{R}}=1+\operatorname{tr} P^{1,1}
$$

для любой вещественной поверхности, где $P^{1,1}$ - примитивная часть в $H^{1,1}$ (которая, фактически, имеет коразмерность 1 в $\left.H^{1,1}\right)$. Следовательно, для $M$-поверхности выполнено равенство

$$
\beta_{1}^{\mathbb{R}}=1+2\left(h^{1,0}+\nu\right)+h^{2,0}+p_{-}^{1,1},
$$

где $p_{-}^{1,1}$ - размерность антиинвариантного подпространства в $P^{1,1}$ относительно действия вешественной структуры. С другой стороны, для поверхностей Мияоки-Яо имеет место равенство

$$
3\left(2+2 h^{2,0}-h^{1,1}\right)=2-4 h^{1,0}+2 h^{2,0}+h^{1,1}
$$

и, таким образом,

$$
h^{1,1}=h^{2,0}+h^{1,0}+1 .
$$

В итоге для максимальной вещественной поверхности Мияоки-Яо выполняется равенство

$$
\beta_{1}^{\mathbb{R}}=h^{1,1}+p_{-}^{1,1}+h^{1,0}+2 \nu .
$$

Из этого равенства вытекает, что либо для всех максимальных вешественных поверхностей Мияоки-Яо с $h^{1,0}=0$ выполнено равенство $p_{-}^{1,1}=\nu=0$ (что было бы удивительно), либо сушествует (максимальная) вешественная поверхность Мияоки-Яо с $h^{1,0}=0$ и $\beta_{1}^{\mathbb{R}}>h^{1,1}$ (что является более вероятным).

Следуюшие предложения показывают, что если максимальные вещественные поверхности Мияоки-Яо сушествуют, то их топология имеет сильные ограничения. Отметим также, что предложение 5.2 дает оценку снизу на $|e(F)-1|$ для всех компонент $F$ вещественной части максимальной вещественной поверхности Мияоки-Яо $X$, в то время как более традиционные результаты дают оценки сверху на $\left|e\left(X_{\mathbb{R}}\right)-1\right|$, где $X_{\mathbb{R}}$ - множество всех вешественных точек многообразия $X$ (см., например, обзор [3]).

ПРЕДЛОЖЕНИЕ 5.1. Не существует максимальных вещественных поверхностей Мияоки-Яо с $h^{2,0} \leqslant 3$.

ДокАЗАТЕльство. Пусть $X$ - максимальная вешественная поверхность Мияоки-Яо. Обозначим через $k$ число связных компонент поверхности $X_{\mathbb{R}}$. Из (5.2) следует, что

$$
2 k-\beta_{1}^{\mathbb{R}}=1+p_{+}^{1,1}-p_{-}^{1,1} .
$$

Подставляя $\beta_{1}^{\mathbb{R}}$ из $(5.5)$ в (5.6) и учитывая $(5.4)$, получаем

$$
2 k=h^{1,1}+h^{1,0}+2 \nu+p_{+}^{1,1}+1
$$


По теореме 5.2 (так как универсальная накрываюшая поверхности $X$ является шаром [10], [14]) $\operatorname{dim} H_{1}(S ; \mathbb{Z} / 2 \mathbb{Z}) \geqslant 3$ для любой связной компоненты $S$ поверхности $X_{\mathbb{R}}$. Следовательно, $\beta_{1}^{\mathbb{R}} \geqslant 3 k$ и из (5.2) и (5.7) следует неравенство

$$
2 p_{-}^{1,1} \geqslant h^{1,1}+h^{1,0}+2 \nu+3 p_{+}^{1,1}+3,
$$

которое эквивалентно неравенствам

$$
h^{1,1} \geqslant h^{1,0}+2 \nu+5 p_{+}^{1,1}+5, \quad h^{2,0} \geqslant 2 \nu+5 p_{+}^{1,1}+4 .
$$

Следовательно, $h^{2,0} \geqslant 4$.

ПРЕДЛОЖЕнИЕ 5.2. Пусть Х - максимальная вещественная поверхность Мияоки-Яо. Тогда по крайней мере три связные компоненты поверхности $X_{\mathbb{R}}$ диффеоморфны сфере с тремя раздутымми точками.

ДоКАЗАТЕЛЬСТво. Обозначим через $k_{3}$ число связных компонент поверхности $X_{\mathbb{R}}$, диффеоморфных сфере с тремя раздутыми точками. Тогда по теореме 5.2

$$
\operatorname{dim} H_{1}(S ; \mathbb{Z} / 2 \mathbb{Z}) \geqslant 4
$$

для всех других связных компонент $S$ поверхности $X_{\mathbb{R}}$. Следовательно, $\beta_{1}^{\mathbb{R}} \geqslant$ $4 k-k_{3}$. Из (5.5) и (5.7) следует, что в этом случае должно быть выполнено следуюшее неравенство:

$$
h^{1,1}+h^{1,0}+2 \nu+p_{-}^{1,1} \geqslant 2 h^{1,1}+2 h^{1,0}+4 \nu+2 p_{+}^{1,1}+2-k_{3},
$$

которое противоречит неравенству $h^{1,1}>p_{-}^{1,1}$, если $k_{3}<3$.

5.4. Поверхности Мияоки-Яо являются квазипростыми в следующем смысле: две вешественные структуры на этих поверхностях сопряжены с помощью автоморфизма тогда и только тогда, когда они сопряжены с помощью некоторого диффеоморфизма ${ }^{2}$. Это утверждение следует из сильной жесткости Мостова и из того, что изометрия компактного гиперболического риманова многообразия, действующая тождественно на фундаментальной групп, является тождественным отображением. (Отметим, что две вещественные структуры являются сопряженными с помощью элемента из Aut, как только они сопряжены с помощью элемента из Kl.)

\footnotetext{
${ }^{2}$ В общем случае вещественная квазипростота деформационного класса многообразий должна означать, что две вещественные структуры являются вещественно деформационно эквивалентными тогда и только тогда, когда они сопряжены при помощи некоторого диффеоморфизма.

Добавление при корректуре. Используя невещественную поверхность, построенную в $\S 4$, либо фальшивые проективные плоскости (см. $\S 5$ ), можно получить многообразия $X$ произвольной размерности $\geqslant 3$, имеющие те же самые свойства, т.е. примеры таких $X$, что $X$ и $\bar{X}$ принадлежат разным связным компонентам пространства модулей. Достаточно рассмотреть произведения этих поверхностей (чтобы получить примеры в произвольной четной размерности) или произведения этих поверхностей и кривой рода больше 1 (в случае нечетной размерности). У тверждение о компонентах пространства модулей будет следовать из хорошо известных свойств отображения Альбанезе и теоремы о жесткости Сиу.

$\Phi$. Катанезе сообщил, что он также построил примеры поверхностей, для которых комплексное сопряжение переставляет компоненты в пространстве модулей этих поверхностей. Его поверхности накрываются бидиском $D \times D \subset \mathbb{C}^{2}$.
} 


\section{Список литературы}

1. Barth W., Peters C., Van de Ven A. Compact Complex Surfaces. N. Y.: Springer-Verlag, 1984.

2. Barthel G., Hirzebruch F., Höfer Th. Geradenkonfigurationen und Algebraische Flöhen. Braunschweig: Friedr. Vieweg \& Sohn, 1987.

3. Дегтярев A., Харламов В. Топологические свойства вещественных алгебраических многообразий // УМН. 2000. Т. 55. № 4. С. 129-212.

4. Grauert H., Remmert R. Komplexe Raüme // Math. Ann. 1958. V. 136. P. 245-318.

5. Hirzebruch $F$. Arrangements of lines and algebraic surfaces // Arithmetics and Geometry. V. II. Prog. Math. № 36. Birkháuser, 1983. P. 113-140.

6. Ishida M.-N. The Irregularities of Hirzebruch's Examples of Surfaces of General Type with $c_{1}^{2}=3 c_{2} / /$ Math. Ann. 1983. V. 262. P. 407-420.

7. Itenberg I. Contre-exemples à la conjecture de Ragsdale // C. R. Acad. Sci. Paris. 1993. V. 317. P. 277-282.

8. Kharlamov V. Variétés de Fano réelles // Sém. Bourbaki. 2000. V. 872.

9. Manetti $M$. On the moduli space of diffeomorphic algebraic surfaces // Invent. Math. 2001. V. 143. P. 29-76.

10. Miyaoka $Y$. On algebraic surfaces with positive index // Classification of algebraic and analytic manifolds. Prog. Math. V. 39. Birkháuser, 1983. P. 281-301.

11. Mumford D. An algebraic surface with $K$ ample, $K^{2}=9, p_{g}=q=0 / /$ Amer. J. Math. 1979. V. 101. P. 233-244.

12. Namba M. Branched coverings and algebraic functions. N.Y.: Longman Scientific \& Technical, 1987.

13. Preissman A. Quelques propriétés globales des espaces de Riemann // Comment. Math. Helv. 1943. V. 15. P. 175-216.

14. Yau S-T. Calaby's conjecture and some new results in algebraic geometry // Proc. Nat. Acad. Sci. USA. 1977. V. 74. P. 1798-1799. 